\title{
On the methods of extending Dirac's equation of the electron to general relativity
}

\author{
By D. MARTIN.
}

(Received 4th June, 1941. Read 7th June, 1941.)

1. Introduction. The problem of extending Dirac's equation of the electron to general relativity has been attacked by many authors, by methods which fall roughly into either of two classes according as the formulation does or does not require the introduction of a local Galilean system of coordinates at each point of space-time. As examples of the former class we mention the methods of Fock (1929) and of Cartan (1938), and as representing the latter class the method described by Ruse (1937). Also, Whittaker (1937) discovered a vector whose vanishing is completely equivalent to the Dirac equations, but this method, unlike the others in the second category, does not apply the Riemannian technique to spinors but only to vectors and tensors derived from these. Now Cartan has denied the possibility of fitting a spinor into Riemannian Geometry if his point of view of spinors is adhered to, and this he argues accounts for the "choquant" properties with which they have been endowed by the geometricians in order to enable them to write down an expression of the usual form for the covariant derivative of a spinor. Consequently, doubt has been cast on the compatibility of the various methods, so in this paper an attempt is made to clarify the matter by working out explicitly the case of the general metric by some of the more important of these methods.

Throughout, the velocity of light in vacuo is taken as unity.

2. Cartan's Method. From Cartan's point of view a spinor with $2^{v}$ components is regarded as a tensor (in the general sense of the term) furnishing a linear representation of degree $2^{\nu}$ of the group of rotations in flat space of $2 v$ or $(2 v+1)$ dimensions, and an important feature of the representation is its bivalency (i.e. to each rotation there correspond two operations on the spinor). When a spinor is simple as all 4-component spinors are, its components can be interpreted geometrically as parameters specifying an isotropic $\nu$-plane in the null 
cone at the appropriate point of the $2 \nu$ - or $(2 \nu+1)$-dimensional space. To every-spinor there corresponds a unique plane or pair of planes according as the space is of odd or even dimensionality. In the latter case there is a unique plane for each of the semi-spinors comprised in the spinor. A spinor is denoted by a column matrix, and a special feature of Cartan's work is that a vector is represented by a certain square matrix. The same symbol is used for the vector and the matrix representing it.

Under a reflection in a prime whose normal is represented by the unit vector $A$, a spinor $\xi$ and a vector $X$ transform to $\xi^{\prime}$ and $X^{\prime}$ where

$$
\xi^{\prime}=A \xi, \quad X^{\prime}=-A X A .
$$

The vector $A$ is ambiguous in sign, and this is the cause of the bivalency referred to above.

Now Cartan has shown that when a vector suffers an infinitesimal parallel displacement, its components referred to an orthogonal ennuple undergo a transformation corresponding to a rotation of the vector. Thus, already knowing the behaviour of a spinor under a rotation, he really determined its behaviour under a parallel displacement, so consequently obtained an expression for its covariant derivative.

Let the metric be given by

$$
d s^{2}=g_{\mu \nu} d x^{\mu} d x^{\nu}, \quad(\mu, \nu=0,1,2,3),
$$

the signature being $(+---)$, and let us choose an orthogonal ennuple defined at each point of space-time, the contravariant components of the unit tangent to the $h$-th member at the point under consideration being denoted by $e_{h \mid}^{a}$. Greek letters denote tensor suffixes and the summation convention applies, while Roman letters denote ennuplet suffixes and any summation is explicitly stated. Then (Eisenhart, 1926, 96)

$$
e_{h^{\prime}}^{a} e_{l, a}=e_{h} \delta_{h l}
$$

where $e_{0}=1, e_{1}=e_{2}=e_{3}=-1$.

The relationship between the coordinate and ennuplet components of a vector is given by

$$
V^{a}=\sum_{h} e_{h} V_{h}^{\prime} e_{h^{\prime}}^{a}
$$

or, by $(2.2)$,

$$
V_{h}=V_{a} e_{h^{\prime}}^{a}
$$


The more general relation corresponding to $(2.3)$ is

$$
V^{a \beta \gamma} \ldots=\sum_{h, k, l \ldots .} e_{h} e_{k} e_{l} \ldots e_{h:}^{a} e_{k}^{\beta} e_{l}^{\gamma} \ldots V_{h k l \ldots} \ldots
$$

Taking the covariant derivatives of both sides of (2.3) with respect to $x^{\sigma}$, we have

$$
V_{, \sigma}^{\alpha}=\sum_{h} e_{h}\left(V_{h, \sigma} e_{h}^{\alpha}+V_{h} e_{h, \sigma}^{a}\right),
$$

and since this vanishes for a parallel displacement, we obtain

$$
\begin{aligned}
\sum_{h} e_{h} e_{h \mid}^{a} \delta V_{h} & =-\sum_{h} e_{h} V_{h} e_{h, \sigma}^{a} d x^{\sigma} \\
& =-\sum_{h, k} e_{h} e_{k} V_{h} e_{h \mid, \sigma}^{a} e_{k !}^{\sigma} d s_{k},
\end{aligned}
$$

where the $d s_{k}$ are the ennuplet components of the infinitesimal displacement considered.

Multiplying throughout by $e_{l, a}$ and summing with respect to $a$, we have by (2.2)

$$
\delta V_{l}=\sum_{h, k} e_{h} e_{k} V_{h} \gamma_{l h k} d s_{k}
$$

where the $\gamma_{l h k}=e_{l, \sigma}^{a} e_{h a} e_{k \mid}^{\sigma}$ are Ricci's coefficients of rotation.

Hence, if (2.6) is written in the form

then

$$
e_{l} \delta V_{l}=\sum_{h} \omega_{h}{ }^{\prime} e_{h} V_{h} \text {, }
$$

and

$$
\begin{aligned}
\omega_{h \cdot}^{l}= & =\sum_{k} e_{k} e_{l} \gamma_{l h k} d s_{k} \\
\omega_{h l} & =-\omega_{l h} .
\end{aligned}
$$

Now (Cartan t. 2, 87), the covariant differential of a 4-component spinor $\left(\begin{array}{l}\xi_{0} \\ \xi_{12} \\ \xi_{1} \\ \xi_{2}\end{array}\right)$ is given by

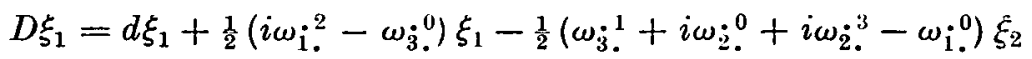

and three similar expressions.

Writing this in the form

$$
D \xi_{1}=D_{0} \xi_{1} e_{0} d s_{0}+D_{1} \xi_{1} e_{1} d s_{1}+D_{2} \xi_{1} e_{2} d s_{2}+D_{3} \xi_{1} e_{3} d s_{3},
$$

putting

$$
d \xi_{1}=\sum_{k} \frac{\partial \xi_{1}}{\partial x^{a}} e_{k} e_{k}^{a} d s_{k},
$$

and using (2.8), we obtain for example

$D_{1} \xi_{1}=\frac{\partial \xi_{1}}{\partial x^{2}} e_{1}^{a}+\frac{1}{2}\left(-\gamma_{031}+i_{\gamma_{121}}\right) \xi_{1}+\frac{1}{2}\left(\gamma_{011}-i_{\gamma_{021}}+\gamma_{131}-i \Upsilon_{231}\right) \xi_{2}$. 
The Dirac wave functions in the form adopted by Weyl (1931) are obtained from the correspondence

$$
\left(\begin{array}{l}
\xi_{0} \\
\xi_{12} \\
\xi_{1} \\
\xi_{2}
\end{array}\right) \rightarrow\left(\begin{array}{c}
\psi_{4} \\
\psi_{3} \\
\psi_{1} \\
-\psi_{2}
\end{array}\right)
$$

Consequently in generalizing the wave equation, $\hat{c} \psi_{1} / \partial x^{1}$ must be replaced by

$$
\frac{\partial \psi_{1}}{\partial x^{a}} e_{1 !}^{a}+\frac{1}{2}\left(-\gamma_{031}+i \gamma_{121}\right) \psi_{1}-\frac{1}{2}\left(\gamma_{011}-i \gamma_{021}+\gamma_{131}-i \gamma_{231}\right) \psi_{2},
$$

with similar expressions for the other derivatives.

These results are easily verified to be in agreement with Fock's expression

$$
\begin{gathered}
D_{l} \psi=\frac{\dot{\partial} \psi}{\dot{c} s_{l}}-\frac{1}{4} \sum_{m, k} e_{k} a_{m} a_{k} \gamma_{m k l l} \psi, \\
\text { where } a_{0}=\left(\begin{array}{cccc}
1 & \cdot & \cdot & \cdot \\
\cdot & 1 & \cdot & \cdot \\
\cdot & \cdot & 1 & \cdot \\
\cdot & \cdot & \cdot & 1
\end{array}\right), a_{1}=\left(\begin{array}{cccc}
\cdot & 1 & \cdot & \cdot \\
1 & \cdot & \cdot & \cdot \\
\cdot & \cdot & \cdot & 1 \\
\cdot & \cdot & 1 & \cdot
\end{array}\right), \\
a_{2}=\left(\begin{array}{cccc}
\cdot & -i & \cdot & \cdot \\
i & \cdot & \cdot & \cdot \\
\cdot & \cdot & \cdot & i \\
\cdot & \cdot & -i & \cdot
\end{array}\right), a_{3}=\left(\begin{array}{cccc}
1 & \cdot & \cdot & \cdot \\
\cdot & -1 & \cdot & \cdot \\
\cdot & \cdot & 1 & \cdot \\
\cdot & \cdot & \cdot & -1
\end{array}\right),
\end{gathered}
$$

and $\psi$ denotes the column matrix $\left(\begin{array}{c}\psi_{1} \\ \psi_{2} \\ \psi_{3} \\ \psi_{+}\end{array}\right)$. Weyl's $\psi$ 's are obtained from the correspondence $\left(\begin{array}{c}\psi_{1} \\ \psi_{2} \\ \psi_{3} \\ \psi_{4}\end{array}\right)_{\text {Fock }} \rightarrow\left(\begin{array}{c}\psi_{3} \\ \psi_{4} \\ -\psi_{2} \\ \psi_{1}\end{array}\right)_{\text {Weyl }}$

Fock derived (2.12) by choosing $D_{l} \psi$ in such a way as to ensure that the 4-vector of charge and current derived from $\psi$ transforms correctly under a parallel displacement. Further, Fock proved that his result is independent of the orthogonal ennuple chosen, and so placed his method in a position not inferior to any of those based on Riemannian Geometry. 
3. Whittaker's Method. This was originally given in the 2 component spinor formalism of van der Waerden, but it is recast here into the 4-component formalism of Cartan, because this treatment requires fewer vectors and also gives more insight into the origin of one of the vectors used. Further, the derivation of a vector involving the derivatives of the components of a spinor is included. This vector (or at least its analogue in 2-component spinor theory) appears to be less well-known than the other vectors associated with a spinor; indeed, I have not yet seen a derivation of it.

Confining ourselves meantime to special relativity, we begin by deriving this vector by a slight extension of a method adopted by Cartan for extracting a 6-vector, a vector, a pseudo-vector, a scalar and a pseudo-scalar from the 16 products $\xi_{r} \xi_{s}^{\prime}$ of the components of two 4-component spinors.

In 4-dimensional space of fundamental form $x^{1} x^{1 \prime}+x^{2} x^{2 \prime}$ (the metric of special relativity is obtained by putting $i x^{1}=x_{1}+i x_{2}$, $i x^{\prime \prime}=x_{1}-i x_{2}, i x^{2}=x_{3}+x_{0}, i x^{2 \prime}=x_{3}-x_{0}$ ), a vector $X$ whose contravariant components are $x^{1}, x^{1 \prime}, x^{2}, x^{2}$ is represented by the matrix

$$
X=\left(\begin{array}{cccc}
\cdot & \cdot & x^{1} & x^{2} \\
\cdot & \cdot & x^{2^{\prime}} & -x^{1^{\prime}} \\
x^{1 \prime} & x^{2} & \cdot & \cdot \\
x^{2 \prime} & -x^{1} & \cdot & \cdot
\end{array}\right)
$$

For example, the matrices corresponding to the coordinate axes are

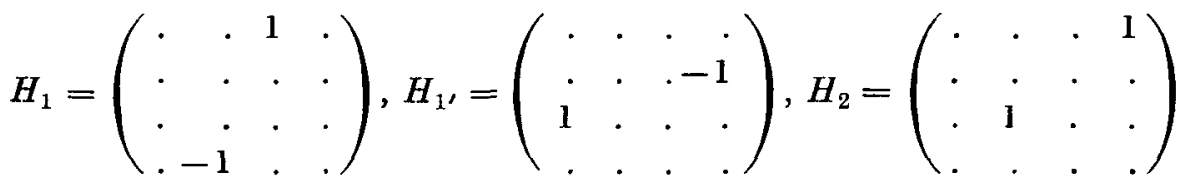

$$
\begin{aligned}
& \text { and } \\
& H_{21}=\left(\begin{array}{cccc}
\cdot & \cdot & \cdot & \cdot \\
\cdot & \cdot & 1 & \cdot \\
\cdot & \cdot & \cdot & \cdot \\
1 & \cdot & \cdot & \cdot
\end{array}\right)
\end{aligned}
$$


then it can be easily verified that for any vector $X$

$$
\boldsymbol{X}^{\prime} C=C X,
$$

where $X^{\prime}$ is the transpose of the matrix $X$.

Since a rotation can be regarded as the product of an even number of reflections, it follows from (2.1) that $\xi^{\prime} C X Y \xi$ where $X, Y$ are any two vectors and $\xi^{\prime}$ is the transpose of $\xi$, is invariant under rotations. Now $\partial / \partial x^{1}, \partial / \partial x^{1 \prime}, \partial / \partial x^{2}, \partial / \partial x^{2 \prime}$ can be regarded as the covariant components of a vector and therefore $\partial / \partial x^{1}, \partial / \partial x^{1}, \partial / \partial x^{2 \prime}$, $\sigma / \partial x^{2}$ as the contravariant components, so that $\xi^{\prime} C X \partial / \partial x \xi$ is an invariant, where $\partial / \partial x$ denotes the matrix

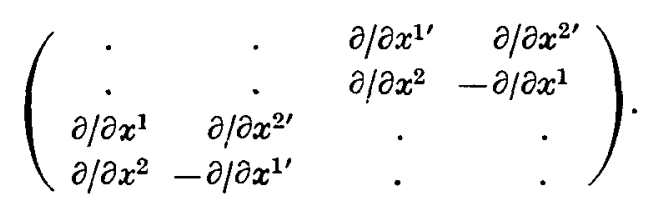

This invariant is bilinear in the $x^{a}$ and $\xi_{r} \delta \xi_{s} / \partial x^{a}$; hence, since $x^{\jmath}, x^{1 \prime}$, $x^{2}, x^{2}$ are contravariant components of a vector, there results the covariant vector

$$
T_{\mu}=\xi^{\prime} C H_{\mu} \partial / \partial x \xi . \quad\left(\mu=1,1^{\prime}, 2,2^{\prime}\right) .
$$

Thus, for example,

and

$$
\begin{gathered}
T_{1}=-\xi_{12}\left(\frac{\partial \xi_{0}}{\partial x^{1}}+\frac{\partial \xi_{12}}{\partial x^{2^{\prime}}}\right)+\xi_{1}\left(\frac{\partial \xi_{1}}{\partial x^{2}}-\frac{\partial \xi_{2}}{\partial x^{1}}\right) \\
T_{1^{\prime}}=\xi_{0}\left(-\frac{\partial \xi_{0}}{\partial x^{2}}+\frac{\partial \xi_{12}}{\partial x^{1^{\prime}}}\right)+\xi_{2}\left(\frac{\partial \xi_{1}}{\partial x^{1^{\prime}}}+\frac{\partial \xi_{2}}{\partial x^{2^{\prime}}}\right) .
\end{gathered}
$$

Changing now to (contravariant) relativistic coordinates $x_{0}, x_{1}, x_{2}, x_{3}$, we have ${ }^{1}$

$$
T_{1}=-i\left(T_{1}+T_{1^{\prime}}\right)
$$

so that

$$
\begin{gathered}
2 T_{1}=-\xi_{0}\left(-\frac{\partial \xi_{12}}{\partial x_{1}}-i \frac{\partial \xi_{12}}{\partial x_{2}}+\frac{\partial \xi_{0}}{\partial x_{3}}+\frac{\partial \xi_{0}}{\partial x_{0}}\right)+\xi_{12}\left(-\frac{\partial \xi_{0}}{\partial x_{1}}+i \frac{\partial \xi_{0}}{\partial x_{2}}-\frac{\partial \xi_{12}}{\partial x_{3}}+\frac{\partial \xi_{12}}{\partial x_{0}}\right) \\
+\xi_{1}\left(-\frac{\partial \xi_{2}}{\partial x_{1}}+i \frac{\partial \xi_{2}}{\partial x_{2}}+\frac{\partial \xi_{1}}{\partial x_{3}}+\frac{\partial \xi_{1}}{\partial x_{0}}\right)+\xi_{2}\left(\frac{\partial \xi_{1}}{\partial x_{1}}+i \frac{\partial \xi_{1}}{\partial x_{2}}+\frac{\partial \xi_{2}}{\partial x_{3}}-\frac{\partial \xi_{2}}{\partial x_{0}}\right) \cdot(3.5)
\end{gathered}
$$

1 There is a slight ambiguity in the symbol $T_{1}$, but it does not persist. 
Now

$$
\begin{aligned}
& \Gamma_{01}=\left(\xi_{12}^{2}+\xi_{1}^{2}-\xi_{2}^{2}-\xi_{0}^{2}\right), \Gamma_{02}=i\left(\xi_{12}^{2}+\xi_{1}^{2}+\xi_{2}^{2}+\xi_{0}^{2}\right), \\
& \Gamma_{03}=-2\left(\xi_{0} \xi_{12}-\xi_{1} \xi_{2}\right), \quad \Gamma_{23}=-i\left(\xi_{12}^{2}-\xi_{1}^{2}+\xi_{2}^{2}-\xi_{0}^{2}\right), \\
& \Gamma_{31}=\left(\xi_{12}^{2}-\xi_{1}^{2}-\xi_{2}^{2}+\xi_{0}^{2}\right), \quad \Gamma_{12}=2 i\left(\xi_{0} \xi_{12}+\xi_{1} \xi_{2}\right),
\end{aligned}
$$

are the components of a 6-vector. This follows immediately from the 6-vector derived from two spinors by making these identical (Cartan 1938, t. 2, 66). Hence (3.5) becomes

$$
T_{1}=\xi_{0} \frac{\partial \xi_{12}}{\partial x_{1}}-\xi_{12} \frac{\partial \xi_{0}}{\partial x_{1}}+\xi_{2} \frac{\partial \xi_{1}}{\partial x_{1}}-\xi_{1} \frac{\partial \xi_{2}}{\partial x_{1}}-\frac{1}{2}\left(\frac{\partial \Gamma_{1 \cdot}^{\cdot 0}}{\partial x_{0}}+\frac{\partial \Gamma_{1}^{* 2}}{\partial x_{2}}+\frac{\partial \Gamma_{1}^{\cdot 3}}{\partial x_{3}}\right) .
$$

Similarly the remaining components can be worked out, and it follows immediately from the form of these that

$$
P_{\mu}=\xi_{0} \frac{\partial \xi_{12}}{\partial x_{\mu}}-\xi_{12} \frac{\partial \xi_{0}}{\partial x_{\mu}}+\xi_{2} \frac{\partial \xi_{1}}{\partial x_{\mu}}-\xi_{1} \frac{\partial \xi_{2}}{\partial x_{\mu}}
$$

$(\mu=0,1,2,3)$ is a covariant vector.

The following vector is also required:

$$
\left.\begin{array}{ll}
A^{0}=-\xi_{0} \xi_{1}-\xi_{12} \xi_{2}, & A^{2}=-i\left(\xi_{0} \xi_{2}-\xi_{12} \xi_{1}\right) \\
A^{1}=\xi_{0} \xi_{2}+\xi_{12} \xi_{1}, & A^{3}=-\xi_{0} \xi_{1}+\xi_{12} \xi_{2}
\end{array}\right\} .
$$

If now the vector $T_{\mu}-i m / \hbar A_{\mu}$, where $m$ and $\hbar$ have their usual physical meanings, is denoted by $\Omega_{\mu}$, and if the spinor components are replaced by the appropriate wave functions (Weyl's), then it follows from (3.5), (3.8), that the components of $\Omega_{\mu}$ are linear combinations of the left-hand sides of the four Dirac equations for the case of zero electromagnetic field. Consequently, since the appropriate determinant is different from zero, the vanishing of $\Omega_{\mu}$ is equivalent to these equations, and this is substantially the first part of Whittaker's result. The main difference is that Whittaker employed the conjugate complexes of the spinor components in addition.. In the treatment given above the divergence term in (3.6) appears automatically, whereas in the 2-component formalism it has to be purposely introduced.

Further, the tensor relationships

and

$$
\begin{aligned}
& 4 A_{a} A^{\beta}=\Gamma_{a \mu} \Gamma^{\beta \mu}, \\
& 2 A_{\sigma} P_{a}=-\Gamma_{\gamma \sigma} A_{, a}^{\gamma}
\end{aligned}
$$

are easily verified. Thus, in general relativity, commencing with $\Gamma_{\mu}$, $A^{\alpha}$ and then $P_{\alpha}$ can be determined by (3.9) and (3.10). $\Gamma_{\mu \nu}$ must be chosen in such a way that the components with respect to an orthogonal ennuple coincide with the special relativity values. $A^{\circ}$ must either 
be chosen in the same way or obtained from (3.9). $P_{a}$, however, can only be obtained from (3.10). In view of the form of $T_{\mu}\left(=P_{\mu}+\right.$ divergence term), the components of $\Omega_{\mu}$ in curved space-time can then be written down. Comparison with the 2-component spinor formalism at this stage shows that the above treatment is much more economical in the use of vectors.

However, in generalizing the wave equation, it is unnecessary to work out the components $\Omega_{\mu}$ explicitly, because by solving (3.10) for $P_{a}$, the expressions by which the derivatives of the spinor components must be replaced can be readily determined: indeed the coefficient of each spinor component in $P_{a}$ gives one such covariant derivative.

By (2.5), (3.10) when expressed in terms of the ennuplet com. ponents of the tensors leads to

$$
\begin{aligned}
2 A_{\sigma} P_{a} & =-\sum_{k, s, l} \Gamma_{k \varepsilon} e_{k i \gamma} e_{s \mid \sigma} e_{k} e_{\delta} e_{l}\left(A_{l} e_{l l}^{\gamma}\right)_{, a} \\
& =-\sum_{k, 8, l} e_{k} e_{s} e_{l} e_{k, \gamma} e_{s \mid \sigma} \Gamma_{k s}\left\{\frac{\partial A_{l}}{\partial x_{\alpha}} e_{l:}^{\gamma}+A_{l} e_{l, a}^{\gamma}\right\} .
\end{aligned}
$$

Multiplying both sides by $e_{t_{1}}^{\sigma}$ and summing with respect to $\sigma$, then by (2.2) and (2.4)

Hence

$$
2 A_{t} P_{a}=-\sum_{k} e_{k} \Gamma_{k t} \frac{\partial A_{k}}{\partial x_{a}}-\sum_{k, i} e_{k} e_{l} e_{k \mid \gamma} e_{l \mid}^{\gamma_{a} a} A_{l} \Gamma_{k t}
$$

$$
\begin{aligned}
2 A_{t} P_{a} e_{t \mid}^{a} & =-\sum_{k} e_{k} e_{t ;}^{a} \Gamma_{k t} \frac{\partial A_{k}}{\partial x_{a}}-\sum_{k, 1} e_{k} e_{l} A_{l} \Gamma_{k t} \gamma_{l k t}, \\
\text { i.e. } \quad 2 A_{t} P_{t} & =-\sum_{k} e_{k} e_{t} \Gamma_{k t} \frac{\partial A_{k}}{\partial s_{t}}-\sum_{k, l} e_{k} e_{l} A_{l} \Gamma_{k t} \gamma_{l k t} .
\end{aligned}
$$

Comparison with $(3.10)^{1}$ shows that if the second term on the right is omitted, the special relativity value of $P_{t}$ will result. Consequently, to obtain the proper expression for $P_{t}$ in general relativity, the quantity

$$
-\frac{1}{2} \sum_{k, l} \frac{e_{k} e_{l} \Gamma_{k t} A_{l} \gamma_{l k t}}{A_{t}}
$$

must be added to the special relativity value.

Using the relation

$$
\Gamma_{m n} A_{p}-\Gamma_{p n} A_{m}=-\Gamma_{p m} A_{n}
$$

these additional terms can be easily worked out, and by considering

In flat space-time ennuplet components become covariant (coordinate) components $[c f .(2.4)]$. 
for example the coefficient of $\xi_{2}$ in $P_{1}$ and replacing $\xi$ 's by $\psi$ 's, it turns out that $c \psi_{1} / c x_{1}$ in the wave equation must be replaced by

$$
\begin{aligned}
D_{1} \psi_{1}=\hat{c} \psi_{1} / \hat{c} x_{a} e_{1 \mid}^{a}+\frac{1}{2}\left(-\gamma_{031}+i \gamma_{121}\right) \psi_{1} \\
-\frac{1}{2}\left(\gamma_{011}-i \gamma_{021}+\gamma_{131}-i \gamma_{231}\right) \psi_{2} .
\end{aligned}
$$

This and the remaining 15 similar expressions agree with those obtained by the previous methods.

4. We pass on now to consider the methods which attempt to use Riemannian Geometry, and follow Ruse's treatment in working out the result. There are, however, a few minor differences in notation.

In this treatment, the components $\psi^{1}, \psi^{2}$ of a 2-component spinor are regarded as parameters specifying a generator of the quadric in which the null cone of the 4-dimensional tangent space $T_{4}$ at the appropriate point of space-time $V_{4}$ intersects the 3-flat $S_{3}$ at infinity. Apart from a normalizing factor, which may be taken as unity, homogeneous coordinates of a point in $S_{3}$ can be regarded as contravariant components of a vector at a point in $V_{4}$. The conjugate components $\psi^{1 *}, \psi^{2 *}$ specify a generator of the other system, and the point of intersection of such a pair of generators represents a null vector whose components can be expressed in terms of the spinor components. Indeed, if the spinor be referred to a real orthogonal ennuple, and if $X^{i}$ denotes the null vector, then

$$
X^{i}=g_{A B *}^{i} \psi^{A} \psi^{B *}, \quad(A, B=1,2),
$$

where

$$
\begin{aligned}
& g_{A B^{*}}^{0}=\frac{1}{\sqrt{ } 2}\left(\begin{array}{rr}
1 & 0 \\
0 & 1
\end{array}\right), \quad g_{A B *}^{1}=\frac{1}{\sqrt{ } 2}\left(\begin{array}{rr}
0 & 1 \\
1 & 0
\end{array}\right), \\
& g_{A B^{*}}^{2}=\frac{1}{\sqrt{ } 2}\left(\begin{array}{rr}
0 & -i \\
i & 0
\end{array}\right), \quad g_{A B *}^{3}=\frac{1}{\sqrt{ } 2}\left(\begin{array}{rr}
1 & 0 \\
0 & -1
\end{array}\right),
\end{aligned}
$$

the summation convention now being applied to all dummy indices. Thus, for example

$$
X^{1}=\frac{1}{\sqrt{ } 2}\left(\psi^{1} \psi^{2 *}+\psi^{2} \psi^{1 *}\right)
$$

Conversely, (4.1) gives

$$
\psi^{A} \psi^{B *}=g_{i}^{A B *} X^{i}
$$

where

$$
\begin{aligned}
& g_{0}^{A B *}=\frac{1}{\sqrt{ } 2}\left(\begin{array}{cc}
1 & 0 \\
0 & 1
\end{array}\right), \quad g_{1}^{A B *}=\frac{1}{\sqrt{ } 2}\left(\begin{array}{rr}
0 & 1 \\
1 & 0
\end{array}\right), \\
& g_{2}^{A B *}=\frac{1}{\sqrt{ } 2}\left(\begin{array}{rr}
0 & i \\
-i & 0
\end{array}\right), \quad g_{3}^{A B *}=\frac{1}{\sqrt{ } 2}\left(\begin{array}{rr}
1 & 0 \\
0 & -1
\end{array}\right) .
\end{aligned}
$$


Spinors are subjected to spin transformations

$$
\psi^{\prime A}=T_{B}^{A} \psi^{B}, \quad\left|T_{B}^{A}\right|=1, \quad(A, B=1,2),
$$

and the group of these complex unimodular transformations is isomorphic with the group of real Lorentz transformations of determinant +1 . Such a transformation can be regarded either as a transformation of one generator into another of the same system or as a change of parametrization of the quadric, and as emphasized by Ruse the choice of the latter alternative makes the space transformations and spin transformations independent of each other. This indeed is the crucial point of the theory, for on it hangs the possibility of writing down an expression of the usual form for the covariant derivative of a spinor. This is taken as

where

$$
\begin{gathered}
\psi_{, a}^{A}=\frac{\partial \psi^{A}}{\partial x^{\alpha}}+\Gamma_{B \alpha}^{A} \psi^{B}, \\
\Gamma_{B a}^{A}=\frac{1}{2} g_{\mu}^{A F *}\left(\frac{\partial g_{B F^{*}}^{\mu}}{\partial x^{\alpha}}+\Gamma_{\nu a}^{\mu} g_{B F^{*}}^{\nu}\right),
\end{gathered}
$$

$\Gamma_{\nu \alpha}^{\mu}$ being an ordinary Christoffel symbol derived from the $g_{\mu \nu}$.

To ensure that the ennuplet components of a vector in curved space-time shall coincide with its covariant components in flat spacetime we now choose $g_{A B *}^{a}$ and $g_{a}^{A B *}$ as follows:

$$
g_{A B *}^{a}=e_{i \mid}^{a} g_{A B *}^{i}, \quad g_{a}^{A B *}=e_{i} e_{i ! a} g_{i}^{A B *},
$$

where the $g$ 's with small Latin indices are given by (4.2) and (4.4), and the $e_{i !}^{a}$ etc., have the same meanings as before.

Now Dirac's equations, in the case of zero electromagnetic field, may be written

$$
\begin{aligned}
& g_{A E *}^{a} \psi_{, a}^{E *}+i_{\kappa \chi_{A}}=0, \quad(A=1,2), \\
& g_{E A *}^{a} \chi_{, a}^{E}-i_{\kappa} \psi_{A}^{*}=0, \quad(A=1,2),
\end{aligned}
$$

where the $\psi$ 's and $\chi$ 's can be identified as certain of Weyl's wave functions, and $\kappa=m /(\hbar \sqrt{ } 2)$.

Considering the first set of equations, (4.5) and (4.6) lead to

$$
e_{i \mid}^{\alpha} g_{A E *}^{i} \frac{\hat{c} \psi^{E *}}{\hat{c} x^{\alpha}}+\frac{1}{2} e_{j} g_{A E *}^{i}\left(g_{j}^{E F *} g_{B F^{*}}^{l}\right)^{*} \gamma_{i j i} \psi^{B *}+i_{\kappa \chi_{A}}=0
$$

Hence, in generalizing the wave equation $\partial \psi^{E *} / \hat{c} x^{i}$ must be replaced by

$$
\frac{\partial \psi^{E *}}{\hat{r} x^{\alpha}} e_{i \mid}^{a}+\frac{1}{2} e_{j} \gamma_{\mid j i} g_{j}^{i E *} g_{F B *}^{l} \psi^{B *}
$$


whence from the correspondence $\left(\begin{array}{c}\psi^{1 *} \\ \psi^{2 *} \\ -\chi^{2} \\ \chi^{1}\end{array}\right) \rightarrow\left(\begin{array}{c}\psi_{1} \\ \psi_{2} \\ \psi_{3} \\ \psi_{4}\end{array}\right)_{\text {Weyl }}$ we obtain expressions for the covariant derivatives, which turn out to be identical with those established by the other methods.

The 2-component spinors used above, each of which corresponds to a generator of the fundamental quadric and therefore to a plane of the null cone are really semi-spinors $(c f . \S 2)$, and the treatment given can only be applied to a 4-component spinor by applying it separately to the two semi-spinors. Furthermore, if $\nu>3$, a semispinor whose components $\left(2^{v-1}\right.$ in number) are all independent, as they would be if they represented wave functions, cannot be simple (Cartan, t. 2, 39); hence, in this case it would be impossible to regard the spin transformations as changes of parametrization. Some other method of securing the independence of space and spin transformations would be required. It seems then that the method just described is possible only because the Dirac wave functions form a spinor with only 4 components.

Nevertheless, a geometrical treatment, in which the 4 wave functions are regarded as forming a single spinor, has been developed by projective relativity methods in a series of papers culminating in that of Veblen, Taub and von Neumann (1934). Here the result is essentially different, and agrees with those derived above only in certain circumstances, and when a certain constant is given the value - 1. Pauli suggested that the extra term otherwise present may be associated in some way with the neutron; in any case, its mere presence indicates a radical difference between this method and the others.

In conclusion, mention must be made of the important paper by Schrödinger (1932) which, though different in interpretation, is formally identical with that of Fock. Schrödinger, following Tetrode, began by generalizing the Dirac matrices $\alpha_{i}$, so that

$$
a_{i} a_{j}+a_{j} a_{i}=2 g_{i j} \text {. }
$$

The paper has the merit of dispelling the idea that the Dirac equations could only be generalized in space-time admitting teleparallelism, and also of paving the way to the methods of Infeld and van der Waerden, Schouten, and Veblen, which form the basis of the paper by Ruse. 
Finally, I wish to thank Prof. E. T. Whittaker for introducing me to the subject of spinors.

\section{REFERENCES.}

Cartan : Leçons sur la Théorie des Spineurs, t. 2 (1938).

Eisenhart: Riemannian Geometry (1926).

Fock : Zeitschrift für Physik, 57 (1929), 261.

Ruse : Proc. Royal Soc. Edinburgh, 57 (1937), 97.

Schrödinger : Sitzungsberichte der Al:ad. Berlin (1932), 105.

Veblen, Taub and von Neumann : Proc. N. A. S., 20 (1934), 383.

Weyl : Theory of Groups and Quantum Mechanics (1931).

Whittaker : Proc. Royal Soc., A, 158 (1937), 38.

The Royal Technical College,

GLaSGOW. 